\title{
Aggregate Queries over Ontologies
}

\author{
Diego Calvanese, Evgeny Kharlamov, Werner Nutt, Camilo Thorne \\ Free University of Bozen-Bolzano \\ Piazza Domenicani, 3 \\ 39100 Bozen-Bolzano, Italy \\ \{calvanese,kharlamov,nutt,cthorne\}@inf.unibz.it
}

\begin{abstract}
Answering queries over ontologies is an important issue for the Semantic Web. Aggregate queries were widely studied for relational databases but almost no results are known for aggregate queries over ontologies. In this work we investigate the latter problem. We propose syntax and semantics for epistemic aggregate queries over ontologies and study query answering for MAX, MIN, COUNT, CNTD, SUM, AVG queries for the ontology language $D L-$ Lite $_{\mathcal{A}}$.
\end{abstract}

\section{Categories and Subject Descriptors}

H.2.3 [Languages]: Query languages; H.4 [Information Systems Applications]: Miscellaneous

\section{General Terms}

Algorithms, Languages, Theory

\section{Keywords}

Aggregate Queries, Incomplete Information, Ontology Languages, Description Logic

\section{INTRODUCTION}

In ontology-based data access (OBDA), typical of the Semantic Web [13], Enterprise Information Integration, and Data Integration [15], ontologies are used to provide a conceptual view over data repositories, and to mediate the access to the information stored therein. Typically, in OBDA, the data stored in the databases (DBs) is assumed to provide only an incomplete account of the domain of interest, and the ontology can be used to overcome such incompleteness. Indeed, an ontology does not only provide the terms through which the information can be accessed, but may also express various forms of constraints over the domain. By means of logical inference over such constraints, new information can be deduced from the explicitly given one, and contribute to the answers to queries posed to the DB through the ontology $[8,9,12,18]$.

Permission to make digital or hard copies of all or part of this work for personal or classroom use is granted without fee provided that copies are not made or distributed for profit or commercial advantage and that copies bear this notice and the full citation on the first page. To copy otherwise, to republish, to post on servers or to redistribute to lists, requires prior specific permission and/or a fee.

ONISW'08, October 30, 2008, Napa Valley, California, USA.

Copyright 2008 ACM 978-1-60558-255-9/08/10 ...\$5.00.
Hence, the OBDA setting is profoundly different from that of ordinary DBs, where information is assumed to be complete, and constraints expressed over the DB schema (e.g., keys and foreign keys) are used only for validating data, but not for query answering. Instead, query answering in OBDA is tightly related to query answering under constraints over incomplete DBs interpreted under the open-world assumption $[6,5]$. In both cases, query answering amounts to computing the so-called certain answers, i.e., those answers that hold in all possible DB states compatible with the data explicitly stored in the $\mathrm{DB}$ and with the constraints (in the ontology or in the DB schema).

Recently, the problem of answering queries over ontologies has been intensively investigated under various assumptions on the language used to express the ontology. One line of research has considered very expressive languages, i.e., significant fragments of OWL-DL ${ }^{1}$, aiming at determining the decidability of query answering, and establishing its computational complexity $[9,10,12,16]$. Of special interest has been characterizing the data complexity of the problem, i.e., the complexity measured solely in the size of the DB, which typically dominates the size of the intensional level of the ontology. It has been shown that for expressive ontology languages such as $\mathcal{S H \mathcal { L }}$, answering unions of conjunctive queries (UCQs) is coNP-complete in data complexity [12, 16]; actually, the coNP lower bound already holds for much simpler languages due to the presence of disjunction [11]. This has motivated the interest in simpler ontology languages, for which query answering has low data complexity. Specifically, the DL-Lite-family of ontology languages has been identified recently [8, 17], which provides an interesting trade-off between expressive power and data complexity of query answering. On the one hand, DL-Lite (and its variants) have sufficient expressive power to capture conceptual modeling languages, such as the Entity-Relationship model or UML Class Diagrams. On the other hand, answering UCQs under the certain answer semantics over a DL-Lite ontology is LOGSPACE in data complexity, and can be reduced to standard query evaluation over the DB storing the extensional information $[8,17]$. This makes it possible to leverage on standard relational technology, not only for storing the data, but also for query evaluation, thus achieving full scalability w.r.t. the size of the data.

Regarding the query language, it is worth noticing that most research so far has considered just conjunctive queries (CQs) or unions thereof (UCQs), corresponding to the (UNION)-SELECT-PROJECT-JOIN fragment of SQL. An excep-

\footnotetext{
${ }^{1}$ http://www.w3.org/2007/OWL/
} 


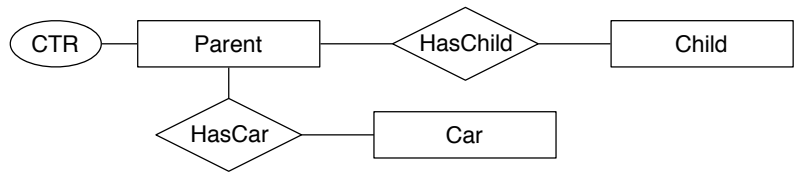

Figure 1: The family ontology.

(a) What is the maximum per child tax reduction explicitly recorded in the data?

(b) How many parents own cars?

(c) What is the total tax reduction of each parent?

Table 1: Example questions involving aggregations.

tion is [7], which allows for "closing" information through the use of an epistemic operator, and then performing firstorder operations. However, such kinds of queries do not allow one to express many natural and interesting information requests, such as those expressed by aggregate queries, like SQL queries using the aggregation functions COUNT, SUM, or AVG. An important aspect of such queries is that the multiplicities of tuples in the answer set over which the aggregation is performed are of importance ${ }^{2}$.

Example 1.1. The ontology shown in Figure 1, rendered as an ER-diagram, models (part of) the information regarding tax payments for families, where the HasChild (resp., HasCar) relation associates Parents with their Children (resp., Cars), and CTR is an attribute representing the per-child tax reduction that a parent has right to (e.g., in Italy, this amount depends on the yearly income). Examples of typical questions over such an ontology are shown in Table 1.

There are only few results related to aggregate queries in a setting of incomplete information [3, 4, 14], and none of them addresses properly the recently introduced ontology languages able to capture conceptual data models, such as those of the DL-Lite family. The main aim of this paper is to fill this gap, by providing the following contributions:

- We show that standard certain answer semantics is not appropriate in general for aggregate queries or for conditional aggregate queries, where part of the query is used to express a condition that should not account for multiplicities in the answer (see Section 4).

- We introduce (conditional) epistemic aggregate queries, and provide for them an alternative, epistemic, semantics that is better suited in the presence of incomplete information (see Section 5).

- For the ontology language $D L-$ Lite $_{\mathcal{A}}$ [17], the most significant representative of the $D L$-Lite family, we investigate conditions on the epistemic aggregate query and the ontology that ensure that certain answers will not necessarily be empty; we develop an algorithm for computing such answers that relies on known algorithms for computing certain answers for non-aggregate queries (see Section 6).

Preliminaries on aggregate queries and on the $D L$-Lite $\mathcal{A}_{\mathcal{A}}$ ontology language are given in Sections 2 and 3, respectively.

${ }^{2}$ Note that in queries expressible in the language of [7], multiplicities are not properly taken into account.

\section{AGGREGATE QUERIES OVER DBs}

In this section we first recall basic notions on conjunctive and aggregate queries in SQL (see also [1] for details). We then recall nested conditional SQL queries, and introduce an extension of rule-based notation to capture such queries.

\subsection{Databases and Conjunctive Queries}

We assume as given a countably infinite domain $\Delta:=$ $\Delta_{O} \cup \Delta_{V}$, partitioned into a domain $\Delta_{O}$ of object constants and a domain $\Delta_{V}$ of values (containing numbers, e.g., $\mathbb{N}$ ). We call tuple any finite sequence $\bar{c}$ of domain elements. We will denote the empty tuple as ().

A term (like $t$ ) is either a variable (like $v, w, x, y, z$ ) or a constant (like $c$ ). A relation name $R$ is a predicate symbol of some arity $n$ (a nonnegative integer). A $D B$ schema $\mathbf{R}$ is a finite set of relation names. An atom is an expression of the form $R(\bar{t})$, where $R$ is a relation name of arity $n$ and $\bar{t}$ is a sequence of $n$ terms. An atom is ground when all its terms are constants.

A condition $\phi$ over $\mathbf{R}$ is a conjunction of atoms with relation names from $\mathbf{R}$, written in the form of a list. We denote by $\operatorname{Var}(\phi)$ the set of variables occurring in condition $\phi$.

A conjunctive query (CQ) over $\mathbf{R}$ is an expression

$$
q(\bar{x}) \leftarrow \phi,
$$

where $q$ is a relation of some arity $n$ that does not occur in $\mathbf{R}$, called head relation, and $\phi$ is over $\mathbf{R}$. The variables in $\bar{x}$ are called distinguished and $\phi$ is called condition of $q$. Distinguished variables should occur in $\phi$. A CQ is boolean when the sequence $\bar{x}$ is empty. As usual, we denote a CQ through its head relation.

A $D B$ instance, or simply $\mathrm{DB}, D$ of $\mathbf{R}$ is a pair $\left(\Delta,{ }^{D}\right)$ where $\Delta$ is the domain and ${ }^{D}$ is an interpretation function over $\mathbf{R}$, that is, a function mapping each relation name $R$ of arity $n$ in $\mathbf{R}$ to a subset $R^{D}$ of $\Delta^{n}$, i.e., to a relation instance. Observe that a DB is a first order logic interpretation of $\mathbf{R}$. We notice that we allow DBs to be infinite, although we consider only finite representations, by means of an ontology (see Section 3), of (sets of) such possibly infinite DBs.

Example 2.1. Consider the DB schema with relations from Figure 1, $\mathbf{R}_{f}:=$ P Parent, CTR, HasCar, HasChild $\}$, where $f$ in $\mathbf{R}_{f}$ stands for "family". A DB $D_{f}$ of $\mathbf{R}_{f}$ is

\begin{tabular}{|c|c|c|c|}
\hline \multirow{2}{*}{$\frac{\text { Parent }}{\text { Name }}$} & & \multicolumn{2}{|l|}{ CTR } \\
\hline & & PName & Amount \\
\hline Luisa & & Luisa & 100 \\
\hline Anna & & Anna & 150 \\
\hline \multicolumn{2}{|l|}{ HasCar } & \multirow{2}{*}{$\begin{array}{l}\text { HasChild } \\
\text { PName }\end{array}$} & \multirow[b]{2}{*}{ CName } \\
\hline PName & CType & & \\
\hline Anna & VwGolf & Anna & Mario \\
\hline Anna & FiatUno & Luisa & Beppe \\
\hline & & Anna & Paolo \\
\hline
\end{tabular}

For convenience, in examples we use attribute names to denote relation positions.

An assignment $\gamma$ over a condition $\phi$ is a function that maps $\operatorname{Var}(\phi)$ to $\Delta$ and each constant in $\phi$ to itself. Assignments are extended to complex syntactic objects like atoms and conditions in the usual way. Whenever $\gamma(\bar{x})=\bar{c}$ we say that $\bar{x}$ is bound to $\bar{c}$. An assignment $\gamma$ satisfies $\phi$ over a DB 
$D$ if, for each atom $R(\bar{t})$ in $\phi$, we have $\gamma(\bar{t}) \in R^{D}$. We denote by $\operatorname{Sat}_{D}(\phi)$ the set of satisfying assignments of $\phi$ over $D$.

The set of answers $q^{D}$ of a CQ $q(\bar{x}) \leftarrow \phi$ over a DB $D$ is defined as $q^{D}:=\left\{\bar{c} \mid \bar{c}=\gamma(\bar{x}), \gamma \in \operatorname{Sat}_{D}(\phi)\right\}$.

\subsection{Aggregate Queries}

We enrich our syntax by considering now the standard SQL aggregation functions max, min, count, cntd, sum, and avg, in the following denoted by $\alpha$. We call an aggregation term any expression of the form count or $\alpha(y)$, where $y$ is called an aggregation variable. We use $\alpha(\bar{y})$ to denote both cases.

An aggregate query (AQ) over $\mathbf{R}$ is a query of the form:

$$
q(\bar{x}, \alpha(\bar{y})) \leftarrow \phi,
$$

where $\bar{x}$ is a (possibly empty) sequence of grouping variables, $\alpha(\bar{y})$ is an aggregation term and $\phi$ is a condition containing $\bar{x}$ and $\bar{y}$. Moreover, $\bar{y}$ does not occur in $\bar{x}$.

Example 2.2. Question (a) in Table 1 gives rise to the following max-query $q_{1}$ over $\mathbf{R}_{f}$ from Example 2.1:

$$
q_{1}(\max (y)) \leftarrow \operatorname{Parent}(x), \operatorname{CTR}(x, y) .
$$

The same query in SQL notation is:

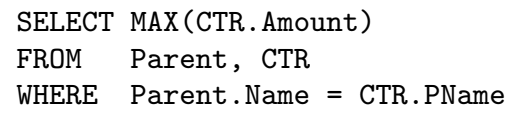

There is no GROUP BY clause, which reflects the fact that in $q_{1}$ there are no grouping variables.

Aggregation functions in SQL are defined over bags $\{\cdot \mid\}$, called groups, of symbolic and numerical values and return a number. Let $D$ be a DB and $\bar{c}$ a tuple. Consider an aggregate query $q(\bar{x}, \alpha(\bar{y})) \leftarrow \phi$. The group $F_{\bar{c}}$ of tuple $\bar{c}$ is defined as the bag

$$
F_{\bar{c}}:=\left\{\gamma(\bar{y}) \mid \gamma \in \operatorname{Sat}_{D}(\phi) \text { and } \gamma(\bar{x})=\bar{c}\right\} .
$$

The set of answers $q^{D}$ of an ACQ $q$ over $D$ is defined as

$$
q^{D}:=\left\{\left(\bar{c}, \alpha\left(F_{\bar{c}}\right)\right) \mid \bar{c}=\gamma(\bar{x}), \text { for some } \gamma \in \operatorname{Sat}_{D}(\phi)\right\} .
$$

Example 2.3. Consider the max-query $q_{1}$ of Example 2.2 and the DB $D_{f}$ of Example 2.1. There are only two satisfying assignments, namely, $\gamma_{1}:=[x /$ Luisa, $y / 100]$ and $\gamma_{2}:=[x /$ Anna, $y / 150]$. Since $q_{1}$ has no grouping variables, the only group we have is for the empty tuple () and $F_{()}=\{100,150\}$. Therefore $q_{1}^{D_{f}}=\left\{\left(\max \left(F_{()}\right)\right)\right\}=$ $\{(\max (\{100,150\}))\}=\{(150)\}$.

\subsection{Conditional Aggregate Queries}

The standard syntax of aggregate queries can only express some SQL aggregate queries. For instance, Question (b) from Table 1 corresponds to the SQL query (over $\mathbf{R}_{f}$ ):

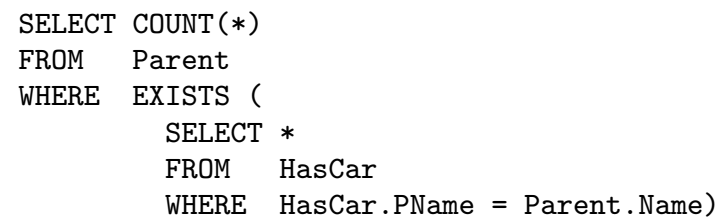

which contains an SQL (sub)query that is nested within an EXISTS condition. Nested SQL subqueries of this kind do not return bags, but a truth value that will be true if and only if there is a tuple satisfying the conditions of the subquery.

To cover this feature, we must extend accordingly the syntax and semantics of aggregate queries. This nesting will be expressed by bracketing atoms in the query's condition.

A conditional aggregate query $q(\mathrm{CAQ})$ is a query of the form:

$$
q(\bar{x}, \alpha(\bar{y})) \leftarrow \phi,[\psi],
$$

where $\phi$ and $\psi$ are conditions, $\bar{x}, \bar{y} \subseteq \operatorname{Var}(\phi)$ and $\operatorname{Var}(\phi) \cap$ $\operatorname{Var}(\psi) \neq \emptyset$. We call $\psi$ a nested condition. As before, we denote queries with their head relation.

We distinguish assignments of $\operatorname{Sat}_{D}(\phi, \psi)$ that give rise to bags of tuples and assignments that do not, i.e., assignments that satisfy nested conditions. We define the latter ones as

$$
\operatorname{Sat}_{D}^{\psi}(\phi):=\left\{\gamma_{\mid \operatorname{Var}(\phi)} \mid \gamma \in \operatorname{Sat}_{D}(\phi, \psi)\right\},
$$

where $\gamma_{\mid \operatorname{Var}(\phi)}$ denotes the restriction of $\gamma$ to $\operatorname{Var}(\phi)$. The group of a tuple $\bar{c}$, denoted $G_{\bar{c}}$, is defined similarly to the group $F_{\bar{c}}$ but using assignments from $\operatorname{Sat}_{D}^{\psi}(\phi)$ :

$$
G_{\bar{c}}:=\left\{\gamma(\bar{y}) \mid \gamma \in \operatorname{Sat}_{D}^{\psi}(\phi) \text { and } \gamma(\bar{x})=\bar{c}\right\} .
$$

We define the set of answers $q^{D}$ of CAQ $q$ over DB $D$ as

$$
q^{D}:=\left\{\left(\bar{c}, \alpha\left(G_{\bar{c}}\right)\right) \mid \bar{c}=\gamma(\bar{x}), \text { for some } \gamma \in \operatorname{Sat}_{D}^{\psi}(\phi)\right\} .
$$

ExAMPLE 2.4. With the new syntax we can express Question (b) from Table 1 as the following CAQ over $\mathbf{R}_{f}$ :

$$
q_{2}(\text { count }) \leftarrow \operatorname{Parent}(x),[\operatorname{HasCar}(x, y)] .
$$

For the DB $D_{f}$ from Example 2.1 we obtain $q_{2}^{D_{f}}=\{(2)\}$.

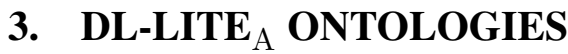

We present now the ontology language on which we base our considerations in the rest of the paper, namely the Description Logic $D L-$ Lite $_{\mathcal{A}}$. This language is one of the most expressive variants of Description Logics (DLs) of the $D L$ Lite family [8], which is a family of DLs that have been developed specifically to handle large amounts of data, possibly stored in relational DBs [17]. DL-Lite $\mathcal{A}$ is also one of the tractable fragments of the upcoming standard OWL $2^{3}$. As usual in DLs, in DL-Lite $e_{\mathcal{A}}$ the universe of discourse is represented in terms of concepts, denoting sets of objects, and roles, denoting binary relations between objects. Additionally, $D L$-Lite $\mathcal{A}_{\mathcal{A}}$ provides mechanisms to deal both with abstract objects (which are instances of concepts) and with data values (such as strings, integers, ...): value-domains denote sets of values, while (concept) attributes ${ }^{4}$ denote binary relations between objects and values. The distinction between objects and values is especially important in the OBDA setting, where one needs to deal with the fact that (abstract) objects are maintained at the level of the ontology, while data values are stored in the underlying DB.

\footnotetext{
${ }^{3}$ http://www .w3.org/2007/OWL/

${ }^{4}$ For simplicity, we do not consider role attributes here.
} 


\subsection{The DL-Lite A $_{\mathrm{A}}$ Language}

In providing the specification of $D L$-Lite $\mathcal{A}$, we use the following notation:

- $A$ denotes an atomic concept, $B$ a basic concept, $C$ a general concept, and $\top_{C}$ the universal concept.

- $E$ denotes a basic value-domain, i.e., the range of an attribute, $F$ a value-domain expression, and $\top_{D}$ the universal value-domain

- $P$ denotes an atomic role, $Q$ a basic role, and $R$ a general role. An atomic role is simply a role denoted by a name.

- $U$ denotes an atomic attribute (or simply attribute), and $V$ a general attribute.

An atomic concept or an atomic value-domain is simply a unary relation name, while an atomic role or an atomic attribute is simply a binary relation name. The syntax of basic and general concepts, value-domains, roles, and attributes is given below. Given an attribute $U$, we call domain of $U$, denoted by $\delta(U)$, the set of objects that $U$ relates to values, and we call range of $U$, denoted by $\rho(U)$, the set of values that $U$ relates to objects. Note that $\delta(U)$ is a concept, whereas $\rho(U)$ is a value-domain.

Formally, the syntax of $D L-$ Lite $_{\mathcal{A}}$ expressions is defined as follows:

$$
\begin{aligned}
& B::=A|\exists Q| \delta(U) \\
& C::=\top_{C}|B| \neg B \mid \exists Q . C \\
& E::=\rho(U) \\
& F:=\top_{D}\left|T_{1}\right| \cdots \mid T_{n} \\
& Q::=P \mid P^{-} \\
& R::=Q \mid \neg Q \\
& V::=U \mid \neg U
\end{aligned}
$$

The semantics of $D L-$ Lite $_{\mathcal{A}}$ is specified, as usual in DLs, in terms of first-order logic interpretations, i.e., DB instances, over the domain $\Delta=\Delta_{O} \cup \Delta_{V}$ (cf. Section 2). All such DB instances agree on the semantics assigned to each valuedomain $T_{i}$. In particular, we assume that $\Delta_{V}$ coincides with the union of the interpretations of all $T_{i}$. Given a DB instance $D=\left(\Delta,{ }^{D}\right)$ interpreting unary and binary atomic relation symbols, the interpretation is extended to complex expressions in the usual way. For lack of space we don't provide here the details of the semantics of the constructs, and refer instead to [17].

\subsection{Knowledge Bases}

A $D$ L-Lite L $_{\mathcal{A}}$ knowledge base $(\mathrm{KB})$, or ontology, $\mathcal{K}=$ $(\mathcal{T}, \mathcal{A})$ is constituted by two components: a TBox $\mathcal{T}$, representing intensional knowledge, and an $\mathrm{ABox} \mathcal{A}$, representing extensional information. The TBox is constituted by a set of assertions of the form:

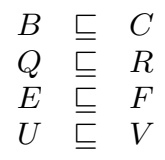

(funct $Q$ )

(funct $U$ ) (concept inclusion assertion)

(role inclusion assertion) (value-domain inclusion assertion) (attribute inclusion assertion)

(role functionality assertion) (attribute functionality assertion)
A concept inclusion assertion expresses that a (basic) concept $B$ is subsumed by a (general) concept $C$. Analogously for the other types of inclusion assertions. A role functionality assertion expresses the (global) functionality of an atomic role. Analogously for an attribute functionality assertion.
Formally, a DB instance $D$ satisfies an inclusion assertion $X \sqsubseteq Y$ if the corresponding set inclusion $X^{D} \subseteq Y^{D}$ holds, and it satisfies a functionality assertion (funct $Q$ ) (resp., (funct $U)$ ), if the relation $Q^{D}$ (resp., $U^{D}$ ) is a function.

A $D L$-Lite $_{\mathcal{A}}$ ABox is a finite set of membership assertions of the form

$$
A(a), \quad D(c), \quad P(a, b), \quad U(a, c)
$$

where $a$ and $b$ are object constants in $\Delta_{O}$, and $c$ is a value in $\Delta_{V}{ }^{5}$. To define its semantics, we specify when a DB instance $D=\left(\Delta,{ }^{D}\right)$ satisfies a membership assertion $\alpha$ in $\mathcal{A}$, written $D \models \alpha$. Namely, $D$ satisfies $A(a)$ if $a \in A^{D}$, satisfies $P(a, b)$ if $(a, b) \in P^{D}$, and satisfies $U(a, b)$ if $(a, b) \in U^{D}$.

$D$ is a model of a $D L$-Lite L $_{\mathcal{A}} \mathrm{KB} \mathcal{K}$ or, equivalently, $D$ satisfies $\mathcal{K}$, written $D=\mathcal{K}$ iff $D$ satisfies all assertions in $\mathcal{K}$. A KB is satisfiable if it has at least one model. A KB $\mathcal{K}$ logically implies an assertion $\alpha$ if all models of $\mathcal{K}$ are also models of $\alpha$. (Similar definitions hold for a TBox $\mathcal{T}$ or ABox A.)

\subsection{Query Answering in DL-Lite}

A query over a $\mathrm{KB} \mathcal{K}$ is a query whose predicates are the atomic concepts, value domains, roles, and attributes of $\mathcal{K}$. The reasoning service we are interested in is query answering: given $\mathcal{K}$ and a query $q(\bar{x})$ over $\mathcal{K}$, return the certain answers $\operatorname{Cert}(q, \mathcal{K})$ to $q(\bar{x})$ over $\mathcal{K}$, i.e., all tuples $\bar{c}$ of elements of $\Delta_{O} \cup \Delta_{V}$ s.t. $\bar{c} \in q^{D}$ for every model $D$ of $\mathcal{K}$.

Note that query answering over an ontology is a form of reasoning over incomplete DBs, and as such, in general, a more complex task than plain query answering over a DB. Indeed, from the results in [8] it follows that, in general, already answering CQs over DL-Lite $\mathcal{A}$ KBs as introduced above, is PTIme-hard in data complexity (i.e., the complexity measured w.r.t. the size of the ABox only). As a consequence, to solve query answering over such KBs, we need at least the power of general recursive Datalog.

However, as shown in [8], the data complexity of answering CQs (and unions thereof) in $D L$ - $_{\text {Lite }} \mathcal{A}$ drops to LogSPACE, provided a suitable restriction is imposed on the interaction between role (resp., attribute) inclusion assertions and functionality assertions. Intuitively, the restriction requires that no functional role or attribute can be specialized, i.e., appears in the left-hand side of an inclusion assertion, when in the right-hand side there is a non-negated role or attribute (we refer to [8] for the details). Moreover, if this condition is satisfied, computing the certain answers of a $\mathrm{CQ}$ with respect to a satisfiable $D L$ - $_{\text {Lite }} \mathcal{A} \mathrm{KB} \mathcal{K}=(\mathcal{T}, \mathcal{A})$ can be reduced, through a process called perfect reformulation, to the evaluation over $\mathcal{A}$ (now viewed as a complete $\mathrm{DB}$ ) of a suitable union of CQs [8]. This query answering technique has also been implemented in the QuOnto system [2], where the ABox is stored directly in a relational $\mathrm{DB}$, containing one relation for each atomic symbol. In the following, we will also assume that the ABox of the ontology over which queries are answered is stored in the form of DB relations, one for each atomic concept, attribute, and role symbol. Note that domains need not be stored explicitly in the DB, since they are actually used only to represent the range of attributes.

${ }^{5}$ We assume to have standard $n a m e s$, i.e., we identify constants with domain elements. 


\section{LIMITATIONS OF CERTAIN ANSWER SEMANTICS}

As we discussed in the previous section, the problem of answering queries over ontologies is a special case of the one of answering queries over sets of DBs. This problem has not yet been studied systematically for aggregate queries.

Lechtenbörger et al. [14] investigated aggregate queries over conditional tables, which are a formalism to specify incomplete DBs, and showed that in this case the answers to an aggregate query can be represented again by a conditional table. Arenas et al. [4] studied aggregate queries over the set of repairs of an inconsistent DB. They introduced a socalled range-semantics, according to which a query is evaluated over each individual instance (that is, each repair), and returns the minimal and the maximal value thus obtained. Afrati and Kolaitis [3] gave a semantics and algorithms for aggregate queries in data exchange, which crucially exploit the specific characteristics of this setting.

As a preparation for the following sections, we will argue that an approach that generalizes the standard certain answer semantics to aggregate queries will not be satisfactory, since in most cases the set of certain answers will be empty.

Example 4.1. Consider the $\mathrm{KB} \mathcal{K}_{1}$ that has an empty TBox and whose ABox is $D_{f}$ from Example 2.1. Because of the open-world semantics of ontologies, $\mathcal{K}_{1}$ expresses that, for instance, Luisa has at least two children, Beppe and Paolo, and that it is unclear whether she has a car.

Recall Question (c) from Table 1. It can be expressed as

$$
q_{3}(x, \operatorname{sum}(y)) \leftarrow \operatorname{CTR}(x, y), \operatorname{HasChild}(x, z) .
$$

In SQL syntax, the query is

$\begin{array}{ll}\text { SELECT } & \text { CTR.PName, SUM (CTR. Amount) } \\ \text { FROM } & \text { CTR, HasChild } \\ \text { WHERE } & \text { CTR.PName = HasChild.PName } \\ \text { GROUP BY } & \text { CTR.PName }\end{array}$

The certain answer semantics for queries over a $\mathrm{KB}$ requires one to compute the intersection of the answer sets over all possible DBs (models) of the KB. Since $\mathcal{K}_{1}$ does not "know" all children of, e.g., Luisa, in different models of $\mathcal{K}_{1}$ the numbers of her children differ and, consequently, the total tax reductions differ. Therefore, the set of certain answers $\operatorname{Cert}\left(q_{3}, \mathcal{K}_{1}\right)$ is empty.

The proposition below generalizes the example to KBs where either the Tbox or ABox are empty.

Proposition 4.2. Let $\mathcal{K}=\langle\mathcal{T}, \mathcal{A}\rangle$ be a $K B$ and $q$ an aggregate query. Then $\operatorname{Cert}(q, \mathcal{K})=\emptyset$ if $\mathcal{T}=\emptyset$ or if $\mathcal{A}=\emptyset$.

Clearly, $\operatorname{Cert}(q, \mathcal{K})=\emptyset$ holds not only in the two extreme cases of the proposition. It holds, intuitively, whenever the TBox does not restrict the instances extending an ABox so much that at least one group has the same aggregate value in each instance.

\section{EPISTEMIC AGGREGATE QUERIES OVER ONTOLOGIES}

We now introduce epistemic aggregate queries and give intuitions why they solve the problems with the certain answers semantics discussed above.

\subsection{Motivation}

Example 5.1. Reconsidering Example 4.1, we notice that certain answer semantics does not exploit the fact that in every instance $D$ that is a model of $\mathcal{K}_{1}$, Luisa has two children for sure, namely Mario and Paolo named in the ABox, and Anna has one child for sure, namely Beppe. Consequently, Luisa will receive a total tax reduction of 300 EUR for Mario and Paolo and Anna one of 100 EUR for Beppe. Clearly, in a specific DB instance, the total reduction for Anna or Luisa may be higher, since the DB instance may contain additional children to the ones explicitly listed in the ABox. If we could tell our query engine that we only want the sum of the known reductions for the known children, then the engine could return as answers to query $q_{3}$ the set $\{$ (Luisa, 300), (Anna, 100)\}.

Note that in this case the answers to the query could be obtained by first computing the certain answers of the conjunctive query

$$
q_{3}^{\prime}(x, y, z) \leftarrow \operatorname{CTR}(x, y), \operatorname{HasChild}(x, z),
$$

then grouping the result according to values of $x$, and finally computing the aggregate $\operatorname{sum}(y)$ for each group (projecting away $z$ ).

The next example shows this semantics is too restrictive.

Example 5.2. Consider a $\mathrm{KB} \mathcal{K}_{2}$, with the following TBox:

$\exists C T R \sqsubseteq \exists$ HasChild, $\quad$ (funct HasChild), (funct CTR),

and consider a DB instance $D$ for $\mathcal{K}_{2}$. The first constraint is satisfied by $D$ if every Parent, with an associated value for the (optional) CTR attribute, has also a child in $D$. The second constraint is satisfied by $D$ if every Parent has at most one child in $D$. The last constraint says that the CTR attribute is functional. One could imagine that DBs satisfying the TBox reflect the situation of a country with a birth control policy where only one child would give the parents a tax reduction. Consequently, a DB instance may contain at most one child for each parent.

Suppose the ABox of $\mathcal{K}_{2}$ is as follows:

\begin{tabular}{l|l}
\multicolumn{2}{l}{ CTR } \\
\hline PName & Amount \\
\hline Luisa & 150 \\
Anna & 100
\end{tabular}

\begin{tabular}{l|l}
\multicolumn{2}{l}{ HasChild } \\
\hline PName & CName \\
\hline Anna & Mario
\end{tabular}

According to the $\mathrm{KB}$, there is one known child of Anna, namely Mario, but no known child of Luisa. However, due to the first constraint, it is known that Luisa has a child, although it is not known who that child is.

If we want to identify the tax reductions that necessarily hold for every DB instance that is a model of $\mathcal{K}_{2}$, it is not sufficient to consider only the known children, as in the previous example, but also which children are known to exist for each person.

Reasoning with the information in the ABox and in the TBox, a query processor could determine that Luisa receives a total reduction of 150 EUR and Anna one of 100 EUR. Actually, in this case $\{($ Luisa, 150), (Anna, 100) $\}$ is the set of certain answers to the query $q_{3}$ over the $\mathrm{KB} \mathcal{K}_{2}$. 
To summarize the intuitions of the semantics for aggregate queries we presented in the examples, in Example 5.1, we (1) calculated certain answers for the query $q_{3}^{\prime}$, the body of which contains the same condition as $q_{3}$, and the distinguished variables of which are $\operatorname{Var}\left(q_{3}\right)$ and (2) aggregated over the certain answers. In Example 5.2, we (1) used certain answers for the grouping variables $\bar{x}$ and the aggregation variable $y$ and (2) applied reasoning over the ontology for the variable $z$, which varies over the children of tax reduction holders, in order to perform the aggregation.

\subsection{Epistemic Aggregate Queries}

In order to capture both of the above cases, we introduce epistemic aggregate queries. An epistemic aggregate query (EAQ) is a query of the form:

$$
q(\bar{x}, \alpha(\bar{y})) \leftarrow \mathbf{K} \bar{x}, \bar{y}, \bar{z} \cdot \phi,[\psi],
$$

where $\bar{z}$ is a possibly empty list of distinct existential variables of $\phi$ and $\psi$ such that $\bar{z}$ is disjoint from $\bar{x}$ and $\bar{y}$.

We call $\mathbf{K}$ an epistemic operator (where the letter " $K$ " stands for "known") and the variables in the list following $\mathbf{K}$ are the epistemic variables, or $\mathbf{K}$-variables, of the query.

EXAmPle 5.3. We introduce query $q_{4}$ as an epistemic variant of query $q_{3}$ in (1)

$$
q_{4}(x, \operatorname{sum}(y)) \leftarrow \mathbf{K} x, y \cdot \operatorname{CTR}(x, y), \operatorname{HasChild}(x, z),
$$

Note that this query has no nested conditions and the only epistemic variables are $x$ and $y$.

\subsection{Semantics of EAQs over Ontologies}

We define the semantics of epistemic aggregate queries for arbitrary KBs.

Consider an EAQ $q$ as in $(2)$ and a $\mathrm{KB} \mathcal{K}$. Let $\bar{w}:=$ $\bar{x} \cup \bar{y} \cup \bar{z}$. A tuple of constants $\bar{c}$ is a known solution for $\bar{w}$ in $\phi, \psi$ over $\mathcal{K}$ if $\bar{c}$ is a certain answer for the query

$$
\operatorname{aux}_{q}(\bar{w}) \leftarrow \phi, \psi
$$

over $\mathcal{K}$. The semantics of $q$ over $\mathcal{K}$ will be defined in such a way that, for every model $D$ of $\mathcal{K}$, the query $q$ is evaluated considering only assignments that map $\bar{w}$ to known solutions. The epistemic certain answers for $q$ over $D$ are then obtained by taking the intersection of the answer sets for all models $D$ of $\mathcal{K}$.

Formally, let $\bar{w}:=\bar{x} \cup \bar{y} \cup \bar{z}$ and $\bar{v}:=\bar{w} \cap \operatorname{Var}(\phi)$, that is, $\bar{w}$ consists of all epistemic variables of $q$, and $\bar{v}$ of all epistemic variables of $\phi$. For a model $D$ of $\mathcal{K}$ we define the set $K_{S a t}, \mathcal{K}(\bar{w} ; \phi, \psi)$ of satisfying $\mathbf{K}$-assignments of $\phi$, $\psi$ over $D$ as

$\operatorname{KSat}_{D, \mathcal{K}}(\bar{w} ; \phi, \psi):=\left\{\gamma \in \operatorname{Sat}_{D}(\phi, \psi) \mid \gamma(\bar{w}) \in \operatorname{Cert}\left(\operatorname{aux}_{q}, \mathcal{K}\right)\right\}$.

Note that all satisfying $\mathbf{K}$-assignments map $\bar{w}$ to known solutions. Analogously to the standard semantics, we define the set $K S a t_{D, \mathcal{K}}^{\psi}(\bar{v} ; \phi)$ of all satisfying $\mathbf{K}$-assignments of $\phi$ over $D$ that respect $\psi$ as

$$
K S a t_{D, \mathcal{K}}^{\psi}(\bar{v} ; \phi):=\left\{\gamma_{\left.\right|_{\operatorname{Var}(\phi)}} \mid \gamma \in \operatorname{KSat}_{D, \mathcal{K}}(\bar{w} ; \phi, \psi)\right\} .
$$

We define the multiset $H_{\bar{d}}$, which we call $\bar{d}$-group, consisting of the satisfying assignments from $\operatorname{KSat}_{D, \mathcal{K}}^{\psi}(\bar{z} ; \phi)$ as follows:

$$
H_{\bar{d}}:=\left\{\gamma(y) \mid \gamma \in K \operatorname{Kat} t_{D, \mathcal{K}}^{\psi}(\bar{z} ; \phi) \text { and } \gamma(\bar{x})=\bar{d}\right\} .
$$

We say that $(\bar{d}, d)$ is a $\mathbf{K}$-answer for an EAQ $q$ over $D$ if there is $\gamma \in K \operatorname{Sat} t_{D, \mathcal{K}}^{\psi}(\bar{v} ; \phi)$ such that

$$
\bar{d}=\gamma(\bar{x}) \quad \text { and } \quad d=\alpha\left(H_{\bar{d}}\right) .
$$

We denote the set of all $\mathbf{K}$-answers for $q$ over a model $D$ of $\mathcal{K}$ as $q(D, \mathcal{K})$.

We define epistemic certain answers for an EAQ $q$ over a knowledge base $\mathcal{K}$, denoted $\operatorname{ECert}(q, \mathcal{K})$, as follows:

$$
\operatorname{ECert}(q, \mathcal{K}):=\bigcap_{D \in \mathcal{K}} q(D, \mathcal{K}) .
$$

EXAmple 5.4. Consider again the epistemic sum-query $q_{4}$ from the knowledge base represented by $\mathcal{K}_{1}$ as in Example 4.1. The certain answers $\operatorname{Cert}\left(q_{3}^{\prime}, \mathcal{K}_{1}\right)$ are

$\{($ Luisa, 100, Beppe), (Anna, 150, Mario), (Anna, 150, Paolo)\}. Then $\operatorname{KSat}_{D, \mathcal{K}}(\bar{w} ; \phi, \psi)$ is $\left\{\gamma_{1}, \gamma_{2}, \gamma_{3}\right\}$ where

$$
\begin{aligned}
\gamma_{1} & :=[x / \text { Luisa, } y / 100, z / \text { Beppe }], \\
\gamma_{2} & :=[x / \text { Anna, } y / 150, z / \text { Mario }], \\
\gamma_{3} & :=[x / \text { Anna, } y / 150, z / \text { Paolo }] .
\end{aligned}
$$

Since $x$ is $q_{4}$ 's grouping variable, this means we get two $\mathbf{K}$ groups, the group of Luisa and the group of Anna, namely, $G_{\text {Luisa }}=\{100\}$ and $G_{\text {Anna }}=\{150,150\}$. Recall that sum returns the sum of the values of the groups. Therefore,

$$
\operatorname{ECert}\left(q_{4}, \mathcal{K}_{1}\right)=\{(\text { Luisa, } 100),(\text { Anna, } 300)\} .
$$

\section{EVALUATING EAQs OVER DL-LITE ${ }_{\mathrm{A}}$ ONTOLOGIES}

As we discussed in Section 4, with the classical approach, for an ACQ $q$ and TBox $\mathcal{T}$, the set of certain answers $\operatorname{Cert}(q,\langle\mathcal{T}, \mathcal{A}\rangle)$ may be empty for every $\operatorname{ABox} \mathcal{A}$. The same can happen for EAQs. We say that an EAQ $q$ is trivial for a TBox $\mathcal{T}$ if for all ABoxes $\mathcal{A}$ it holds that $\operatorname{ECert}(q,\langle\mathcal{T}, \mathcal{A}\rangle)=\emptyset$. We say that a CAQ of the form (2) is coherent with $\mathcal{T}$ if the condition $\phi, \psi$ is satisfiable together with $\mathcal{T}$. Obviously, every query that is non-coherent with $\mathcal{T}$ is trivial for $\mathcal{T}$. In this section, we consider only coherent pairs of queries and $D L$-Lite $\mathcal{A}$ TBoxes, and identify conditions under which these queries are non-trivial for the TBox. We also present algorithms for computing epistemic certain answers for such queries.

In the following we consider a generic EAQ $q$ of form (2).

\subsection{General Algorithm}

We introduce now an algorithm that we will use as the basis for computing epistemic certain answers $\operatorname{ECert}(q, \mathcal{K})$ for the various aggregation functions $\alpha$. We denote the algorithm GA (for General Algorithm). GA takes as input an EAQ $q$ and a KB $\mathcal{K}$, and computes a set of tuples $O=\mathrm{GA}(q, \mathcal{K})$.

We denote the sequence of non-distinguished epistemic variables of $\phi$ as $\bar{z}^{\phi}$. In the algorithm, we denote a predicate (view) of arity $|\bar{x} \cup \bar{y} \cup \bar{z}|$ that "stores" the tuples of certain answers $\operatorname{Cert}\left(a u x_{q}, \mathcal{K}\right)$ for $a u x_{q}$ defined in (3) as $\operatorname{Cert}\left(a u x_{q}, \mathcal{K}\right)$, consequently, $\operatorname{Cert}\left(a u x_{q}, \mathcal{K}\right)(\bar{x}, \bar{y}, \bar{z})$ is an atom with this predicate name. We also denote as qo (resp., $\mathrm{q}_{1}$ ) a predicate (view) that "stores" outputs of $q_{0}$ (resp., $q_{1}$ ).

The algorithm GA is defined in Figure 2 and it basically applies two steps on the tuples in $\operatorname{Cert}\left(a u x_{q}, \mathcal{K}\right)$ : (i) it projects them on components corresponding to epistemic variables of $\phi$ and (ii) it aggregates over the result. 
We say that $q$ has a uniform grouping w.r.t. $\mathcal{T}$ if for every ABox $\mathcal{A}$ and every $\bar{d} \in q^{\prime \operatorname{Cert}\left(\operatorname{ans}_{q},\langle\mathcal{T}, \mathcal{A}\rangle\right)}$, there exists a multiset $M$ such that for every DB instance $D$ compatible with $\langle\mathcal{T}, \mathcal{A}\rangle$, there is a natural number $k$ such that $H_{\bar{d}}=k * M$ (where $H_{\bar{d}}$ defined as in Equation (4)).

Theorem 6.6 (Uniform Grouping). Let $q$ be a coherent epistemic avg-query and $\mathcal{T}$ a DL-Lite $\mathcal{A}$ TBox. If $q$ has a uniform grouping w.r.t. $\mathcal{T}$, then $q$ is non-trivial for $\mathcal{T}$.

We now identify a syntactic conditions on $q$ and $\mathcal{T}$ that guarantees that $q$ has a uniform grouping w.r.t. $\mathcal{T}$, and, hence, is non-trivial for $\mathcal{T}$. We say that an EAQ $q$ is decomposable for $\mathcal{T}$ if $\phi$ can be partitioned into $\phi_{1}, \phi_{2}$ such that:

- $y$ occurs only in $\phi_{1}$,

- all variables in $\phi_{1}$ are restricted by $\mathcal{T}$,

- $\operatorname{Var}\left(\phi_{1}\right) \cap \operatorname{Var}\left(\phi_{2}\right) \subseteq \bar{x}$.

Theorem 6.7 (Decomposable Average Queries). Let $\mathcal{T}$ be a DL-Lite $\mathcal{A}$ TBox and $q$ an epistemic avg-query. If $q$ is decomposable for $\mathcal{T}$, then

- $q$ has uniform grouping w.r.t. $\mathcal{T}$ and

- $\operatorname{ECert}(q,\langle\mathcal{T}, \mathcal{A}\rangle)=G A(q,\langle\mathcal{T}, \mathcal{A}\rangle)$, for every $A B$ ox $\mathcal{A}$.

\section{CONCLUSIONS AND FUTURE WORK}

We have investigated the problem of answering aggregate queries, for which multiplicities in the computed answers need to be taken into account, over ontologies, i.e., in a setting of incomplete information. We have shown that standard certain answer semantics is not appropriate in this setting, and have proposed an alternative semantics based on the notion of epistemic aggregate query (EAQ). For the case of ontologies expressed in DL-Lite A $_{\mathcal{A}}$, we have provided several conditions on the form of the EAQ and of the ontology that guarantee that answers exist and can be computed. In all the identified cases, the computation can be reduced to a standard computation of certain answers for non-aggregate queries; hence, certain answers to EAQs ca be computed in LOGSPACE as is the case for CQs over DL-Lite $\mathcal{A}_{\mathcal{A}}$-knowledge bases. As future work, we plan to extend our results to more expressive ontology languages.

\section{Acknowledgements}

This research has been partially supported by FET project TONES (Thinking ONtologiES), funded within the EU 6th Framework Programme under contract FP6-7603, and by the PRIN 2006 project NGS (New Generation Search), funded by MIUR.

\section{REFERENCES}

[1] S. Abiteboul, R. Hull, and V. Vianu. Foundations of Databases. Addison Wesley Publ. Co., 1995.

[2] A. Acciarri, D. Calvanese, G. De Giacomo, D. Lembo, M. Lenzerini, M. Palmieri, and R. Rosati. QuOnto: Querying OnTologies. In Proc. of the 20th Nat. Conf. on Artificial Intelligence (AAAI 2005), pages 1670-1671, 2005.

[3] F. Afrati and P. Kolaitis. Answering aggregate queries in data exchange. In Proc. of the 27th ACM SIGACT SIGMOD SIGART Symp. on Principles of Database Systems (PODS 2008), 2008.
[4] M. Arenas, L. E. Bertossi, J. Chomicki, X. He, V. Raghavan, and J. Spinrad. Scalar aggregation in inconsistent databases. Theoretical Computer Science, 296(3):405-434, 2003.

[5] A. Calì, D. Calvanese, G. De Giacomo, and M. Lenzerini. Data integration under integrity constraints. Information Systems, 29:147-163, 2004.

[6] A. Calì, D. Lembo, and R. Rosati. On the decidability and complexity of query answering over inconsistent and incomplete databases. In Proc. of the 22nd ACM SIGACT SIGMOD SIGART Symp. on Principles of Database Systems (PODS 2003), pages 260-271, 2003.

[7] D. Calvanese, G. De Giacomo, D. Lembo, M. Lenzerini, and R. Rosati. EQL-Lite: Effective first-order query processing in description logics. In Proc. of the 20th Int. Joint Conf. on Artificial Intelligence (IJCAI 2007), pages 274-279, 2007.

[8] D. Calvanese, G. De Giacomo, D. Lembo, M. Lenzerini, and R. Rosati. Tractable reasoning and efficient query answering in description logics: The DL-Lite family. J. of Automated Reasoning, 39(3):385-429, 2007.

[9] D. Calvanese, G. De Giacomo, and M. Lenzerini. On the decidability of query containment under constraints. In Proc. of the 17th ACM SIGACT SIGMOD SIGART Symp. on Principles of Database Systems (PODS'98), pages 149-158, 1998.

[10] D. Calvanese, G. De Giacomo, and M. Lenzerini. Conjunctive query containment and answering under description logics constraints. ACM Trans. on Computational Logic, 9(3):22.1-22.31, 2008.

[11] F. M. Donini, M. Lenzerini, D. Nardi, and A. Schaerf. Deduction in concept languages: From subsumption to instance checking. J. of Logic and Computation, 4(4):423-452, 1994.

[12] B. Glimm, I. Horrocks, C. Lutz, and U. Sattler. Conjunctive query answering for the description logic $\mathcal{S H \mathcal { I }}$. J. of Artificial Intelligence Research, 31:151-198, 2008.

[13] J. Heflin and J. Hendler. A portrait of the Semantic Web in action. IEEE Intelligent Systems, 16(2):54-59, 2001.

[14] J. Lechtenbörger, H. Shu, and G. Vossen. Aggregate queries over conditional tables. J. of Intelligent Information Systems, 19(3):343-362, 2002.

[15] M. Lenzerini. Data integration: A theoretical perspective. In Proc. of the 21st ACM SIGACT SIGMOD SIGART Symp. on Principles of Database Systems (PODS 2002), pages 233-246, 2002.

[16] M. Ortiz, D. Calvanese, and T. Eiter. Data complexity of query answering in expressive description logics via tableaux. J. of Automated Reasoning, 41(1):61-98, 2008.

[17] A. Poggi, D. Lembo, D. Calvanese, G. De Giacomo, M. Lenzerini, and R. Rosati. Linking data to ontologies. J. on Data Semantics, X:133-173, 2008.

[18] R. Rosati. The limits of querying ontologies. In Proc. of the 11th Int. Conf. on Database Theory (ICDT 2007), volume 4353 of Lecture Notes in Computer Science, pages 164-178. Springer, 2007. 